Journal of Advanced Research in Fluid Mechanics and Thermal Sciences

\title{
Statistical and Simulation Analysis on Dimple Configurations Performance of Heat Dissipation
}

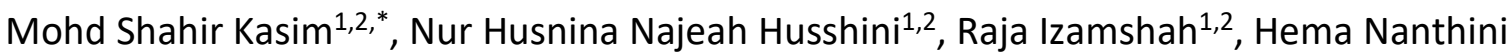 \\ Ganesan², Muhamad Ammar Farhan Maula Mohd Azam², Mohammad Shah All Hafiz ${ }^{1,2}$, Ghazali \\ Omar $^{1}$, Mohd Al Hafiz Mohd Nawi ${ }^{3}$ \\ 1 Advanced Manufacturing Centre, Universiti Teknikal Malaysia Melaka, 76100, Hang Tuah Jaya, Melaka, Malaysia \\ 2 Fakulti Kejuruteraan Pembuatan, Universiti Teknikal Malaysia Melaka, 76100, Hang Tuah Jaya, Melaka, Malaysia \\ 3 Faculty of Mechanical Engineering Technology, Universiti Malaysia Perlis 02100 Padang Besar, Perlis, Malaysia
}

ARTICLE INFO ABSTRACT

\section{Article history:}

Received 11 April 2021

Received in revised form 18 July 2021

Accepted 20 July 2021

Available online 26 August 2021

\section{Keywords:}

Heat transfer enhancement technique; passive method; heat exchanger; Nusselt number

\begin{abstract}
This paper presents an investigation on cooling effect and flow structure of the spherical dimple configuration during air flow on the Aluminium surface. It is prominently known that applying dimples profile causes an enhancement in heat transfer over a plain surface. A three level of Box-Behnken response surface methodology was performed to find the correlation between the input and output variables. A total of 17 different combinations of these inputs were performed throughout the experiment. The variable inputs to be investigated namely: dimple diameter of $10-14 \mathrm{~mm}$, dimple orientation angle of $60^{\circ}-90^{\circ}$, and airflow velocity of 16 - $18 \mathrm{~m} / \mathrm{s}$ to observe the response on the cooling time. The Aluminium block was heated to $60^{\circ} \mathrm{C}$ and cooled down by air flow at room temperature. The ANOVA was used to identify the significant effect of each parameter. CFD software was used as a simulation tool to analyze the flow structure and Reynolds number that associate with the heat transfer rate to support the statistical findings. Based on the result, all the input parameters are found to be significantly dominated by air flow velocity. Staggered arrangement dimple profile surface improves cooling effect by $63 \%$ over the plain flat surface. The increment in Reynolds number will increase the heat transfer which then shortening the cooling time.
\end{abstract}

\section{Introduction}

The constant demand for enhanced heat dissipated product has led to the development of dimple profile surface. It has been studied extensively on the part design which allows heat energy to be transferred easily from one point to another due to the temperature difference [1]. Heat transfer plays a crucial role in many engineering applications such as in the automotive, petrochemical, aerospace and electronics industries. There are many researchers conducted on heat transfer enhancement techniques. These techniques can be categorized into active, passive and compound.

\footnotetext{
* Corresponding author.

E-mail address: shahir@utem.edu.my
}

https://doi.org/10.37934/arfmts.86.2.7490 
In the active method, energy or power such as the electromagnetic field, cams, and plungers are outsourced to improve heat transfer performance. Meanwhile, in the passive method, any external power is not required. The heat is enhanced through surface modification. The compound method consists of the combination of passive and active methods. Since passive method is simpler than active method, it is more popular to be employed in many fields [2-3].

The suggested techniques in passive methods include rib turbulators, pin fins, dimpled surfaces, and protrusion surfaces. The modification of the fabricated surface was made to enhance heat transfer rate [4-5]. The dimple method is one of the best strategies to be considered compared to the others. By applying dimple profile on the flat surface, it will not only increase the heat transfer rate, but also lower the pressure drop penalties [6]. It was initiated by Kuethe [8] that suggested the addition of dimple profile on a flat surface. His study shows that dimpled surfaces could stimulate turbulent mixing flow as to improved heat transfer.

It was supported by Beves et al., [9] who studied the flow structure numerically and experimentally in a two-dimensional spherical cavity on a flat surface. They found that adding dimple profile on a flat surface enhances the heat transfer rate with minimum pressure drop. Study by Paranjape et al., [10] reported the dimpled surface method is better than protruding methods for heat dissipation with less flow resistance.

The key factor for the great improvement in the dimpled heat dissipation method is the thermal boundary layer characteristic, which gets pulled by the cold fluid of the heat sink and is in contact with a high temperature surface [11]. The heat transfer rate of dimpled profile is higher due to the formation of vortex forms and vertical fluid which flows downstream to the flat surface. The mainstream boundary layer is then separated by the dimple profiles, and a recirculation zone is formed at the upstream side inside the dimples. In the downstream of the dimple, the separated mainstream flow reattaches to create a twin vortex [11-12]. This phenomenon significantly enhanced heat dissipation by increasing the heat transfer coefficient. Ligrani et al., [14] investigated on correlation heat transfer coefficients and aerodynamic resistance on surfaces of various physical constructions. They concluded that the heat transfer coefficient increases as the surface area increases, since it generates a huge number of vortex pairs, which stimulates a strong secondary vortex pair.

The geometrical configurations also need to be considered in dimple profile design. Dimple depth-to-diameter ratio, channel height-to-dimple diameter ratio, and dimple spacing have been investigated massively. Study by Kathkhaw et al., [15] shows the higher the dimple depth on a flat surface, the higher the heat transfer improvement. The shorter gaps applied between each dimple on a flat surface have a great impact on heat dissipation augmentation. Bi et al., [16] reported the effect of the dimple profile arrangement on heat transfer augmentation. A tight-spaced dimple arrangement results in higher heat transfer. The dimple diameter should be designed within a standard range, where the diameter cannot be too large or too small. The standard range for the dimple diameter over the height of the control volume for a single dimple is around $0.4-0.64 \mathrm{~mm}$. The dimple geometric size with a deeper and larger diameter allows for greater and more frequent heat dissipation. Deeper dimple also increases the amount of turbulent production and flow [17].

An experimental study was conducted by Mahmood and Ligrani [18] on the influence of dimple and temperature ratio, Reynolds number, and flow structures in the heat transfer. They observed the Reynolds number $(600-11,000)$ and dimple depth-to-dimple diameter ratio $(0.20,0.25,0.5$ and 1.00). It was noticed that the vortex pair sometimes shed from the dimples. This phenomenon leads to local Nusselt number augmentations. Another study about flow structure due to dimple depressions on a channel surface revealed that the changes in dimple depth might influence the flow structure. The increase in dimple depth with respect to dimple diameter may result in greater fluid 
flow. The flow expelled from the dimple might form stronger vortices [19]. A study by Coy and Danczyk [20] concluded that the dimple depth-to-dimple diameter ratio has a significant effect on heat transfer enhancement. The heat transfer enhancement factor gradually increases from 1.4 to 1.8 when the ratio of dimple depth-to-dimple diameter increases from 0.111 to 0.333 .

A study related to dimple arrangement was conducted by Katkhaw et al., [15] on a flat surface. They observed heat transfer of flat surface with ellipsoidal dimple. A dimple profile with a staggered arrangement on a flat surface is more effective than the inline arrangement. For the staggered arrangement and inline arrangement, the heat transfer coefficient improved $21.7 \%$ and $15.8 \%$ respectively than flat plate. It was supported by Patil and Deshmukh [21], showing that the staggered dimple has $66 \%$ better thermal performance factor compared to the dimple with an inline arrangement. The heat transfer coefficient increases as the Reynolds number increases. The heat dissipation enhancement was much better by 2.5 times compared to a flat surface [22]. Another researcher group experimentally investigated the features of heat transfer and fluid flow of spherical dimples at various angles of orientation. They concluded that the heat transfer coefficient and Nusselt number increase with dimples of various orientations compared to a flat surface [23]. An experimental study of heat transfer augmentation with a spherical dimpled surface result concluded that the dimple in a staggered arrangement showed better performance in terms of heat transfer coefficients, Nusselt number, and thermal performance factors compared to the dimple with an inline orientation. The greater vortex formation leads to better thermal performance in the staggered arrangement of dimples [24].

Based on past studies, a general conclusion that can be drawn is dimpled surface on smooth surface shows a significant improvement in heat transfer. However, the correlation between heat transfer, airflow velocity, dimple orientation, dimple diameter, and cooling time less to be reported. The present work to report on these correlations.

\section{Methodology}

\subsection{Experimental Setup}

The investigated variable inputs are dimples diameter, dimples orientation, and airflow velocity. The output responses are cooling time, heat transfer rate, and flow structure. Aluminium 6061 block with the size of $135 \mathrm{~mm} \times 100 \mathrm{~mm} \times 30 \mathrm{~mm}$ was used throughout the experiment. The material selection is due to the fact that it is easy to be machined and possesses good heat conductivity of 202.4 W/mK. The dimple profile on the workpiece was fabricated by using CNC milling machine.

Details of dimple configuration and airflow velocity are shown in Table 1. A two-dimensional schematic view of the core region of Aluminium block with varying diameters and orientation of dimple profiles are depicted in Figure 1, Figure 2, and Figure 3. Figure 4 shows the cross-sectional view staggered arrays of the dimple profiles. The dimple depth ratio to diameter is 0.5 . The samples were tested in wind tunnel dimension (150 $\mathrm{mm} \times 150 \mathrm{~mm} \times 450 \mathrm{~mm}$ ).

Table 1

The selected dimple configurations and airflow velocity

\begin{tabular}{lllllll}
\hline $\begin{array}{l}\text { Dimple } \\
\text { Diameter, } \mathrm{d} \\
(\mathrm{mm})\end{array}$ & $\begin{array}{l}\text { Dimple } \\
\text { Orientation }\end{array}$ & $\begin{array}{l}\text { Height to } \\
\text { diameter } \\
\text { ratio, } \mathrm{H} / \mathrm{d}\end{array}$ & $\begin{array}{l}\text { Dimple } \\
\text { depth to } \\
\text { diameter } \\
\text { ratio, } \mathrm{h} / \mathrm{d}\end{array}$ & $\begin{array}{l}\text { Ratio of } \\
\text { horizontal } \\
\text { intervals }(\mathrm{dx}) \\
\text { to diameter, } \\
\mathrm{dx} / \mathrm{d}\end{array}$ & $\begin{array}{l}\text { Ratio of } \\
\text { vertical } \\
\text { intervals }(\mathrm{dy}) \\
\text { to diameter, } \\
\mathrm{dy} / \mathrm{d}\end{array}$ & $\begin{array}{l}\text { Air } \\
\text { velocity } \\
(\mathrm{m} / \mathrm{s})\end{array}$ \\
\hline $10,12,14$ & $60^{\circ}, 75^{\circ}, 90$ & $2.1,2.5,3.0$ & 0.5 & $1.25-1.75$ & $1.25-1.75$ & $16-18$ \\
\hline
\end{tabular}




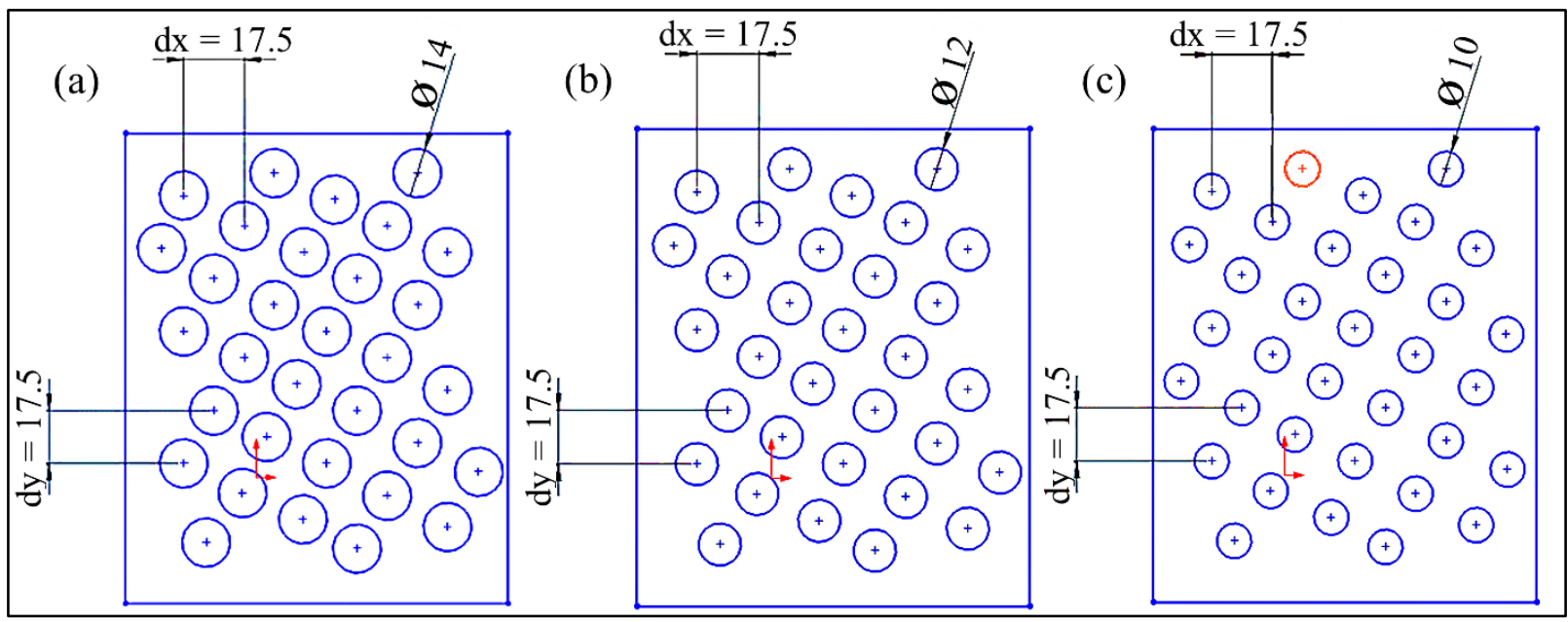

Fig. 1. Dimple Orientation of $60^{\circ}$ (a) Diameter of $14 \mathrm{~mm}$ (b) Diameter of $12 \mathrm{~mm}$ (c) Diameter of $10 \mathrm{~mm}$

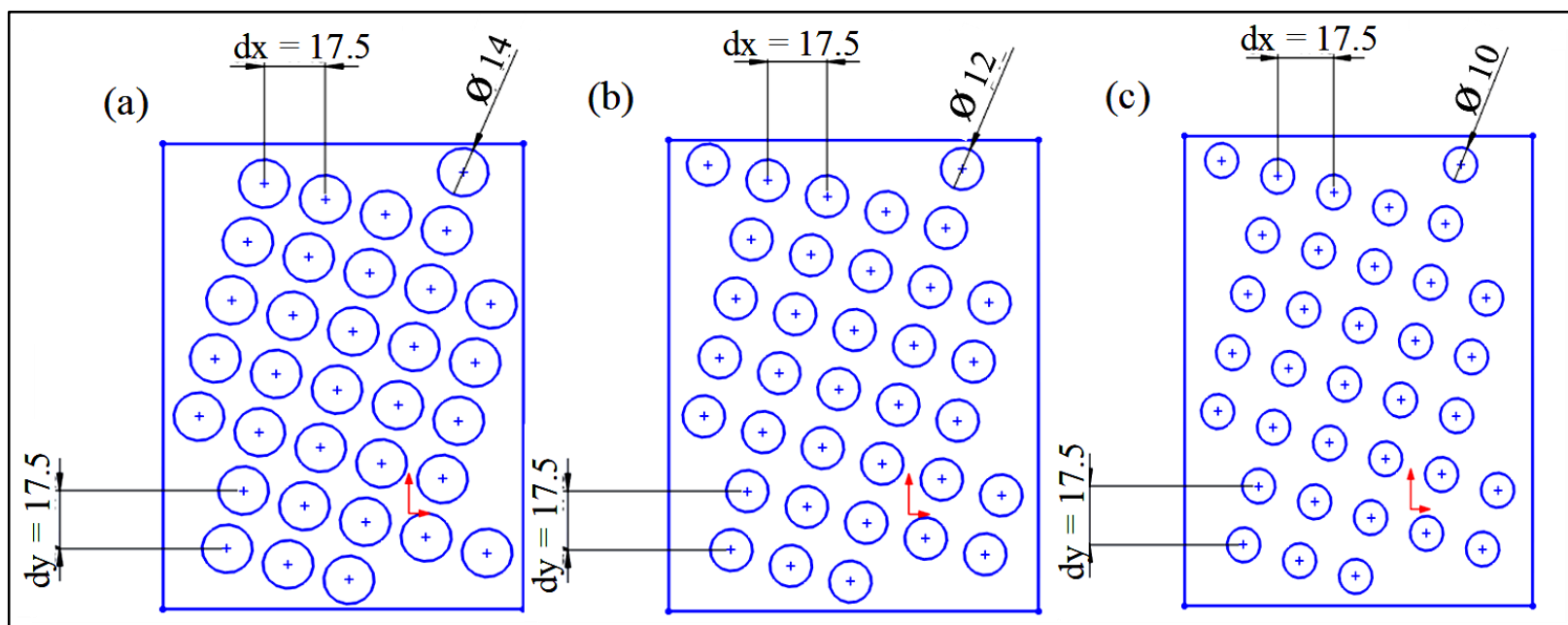

Fig. 2. Dimple Orientation of $75^{\circ}$ (a) Diameter of $14 \mathrm{~mm}$ (b) Diameter of $12 \mathrm{~mm}$ (c) Diameter of $10 \mathrm{~mm}$

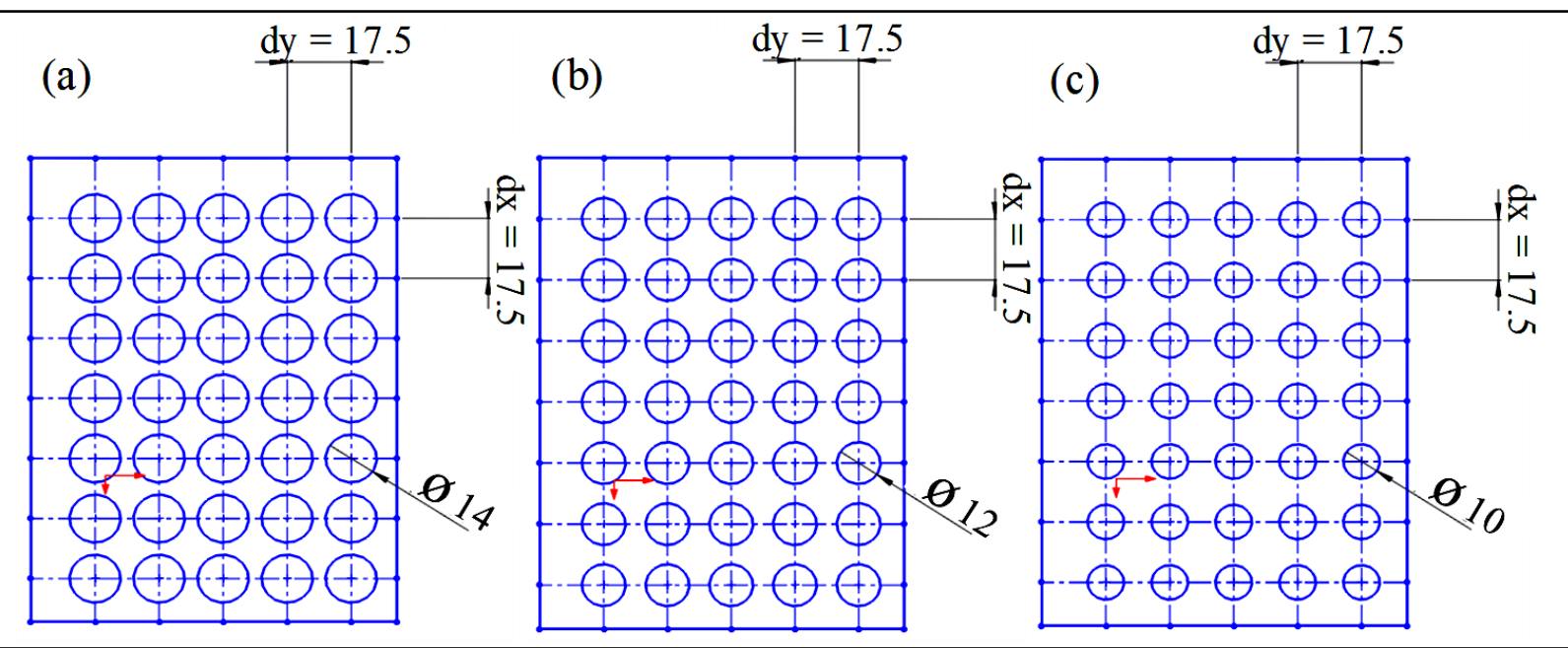

Fig. 3. Dimple Orientation of $90^{\circ}$ (a) Diameter of $14 \mathrm{~mm}$ (b) Diameter of $12 \mathrm{~mm}$ (c) Diameter of $10 \mathrm{~mm}$ 


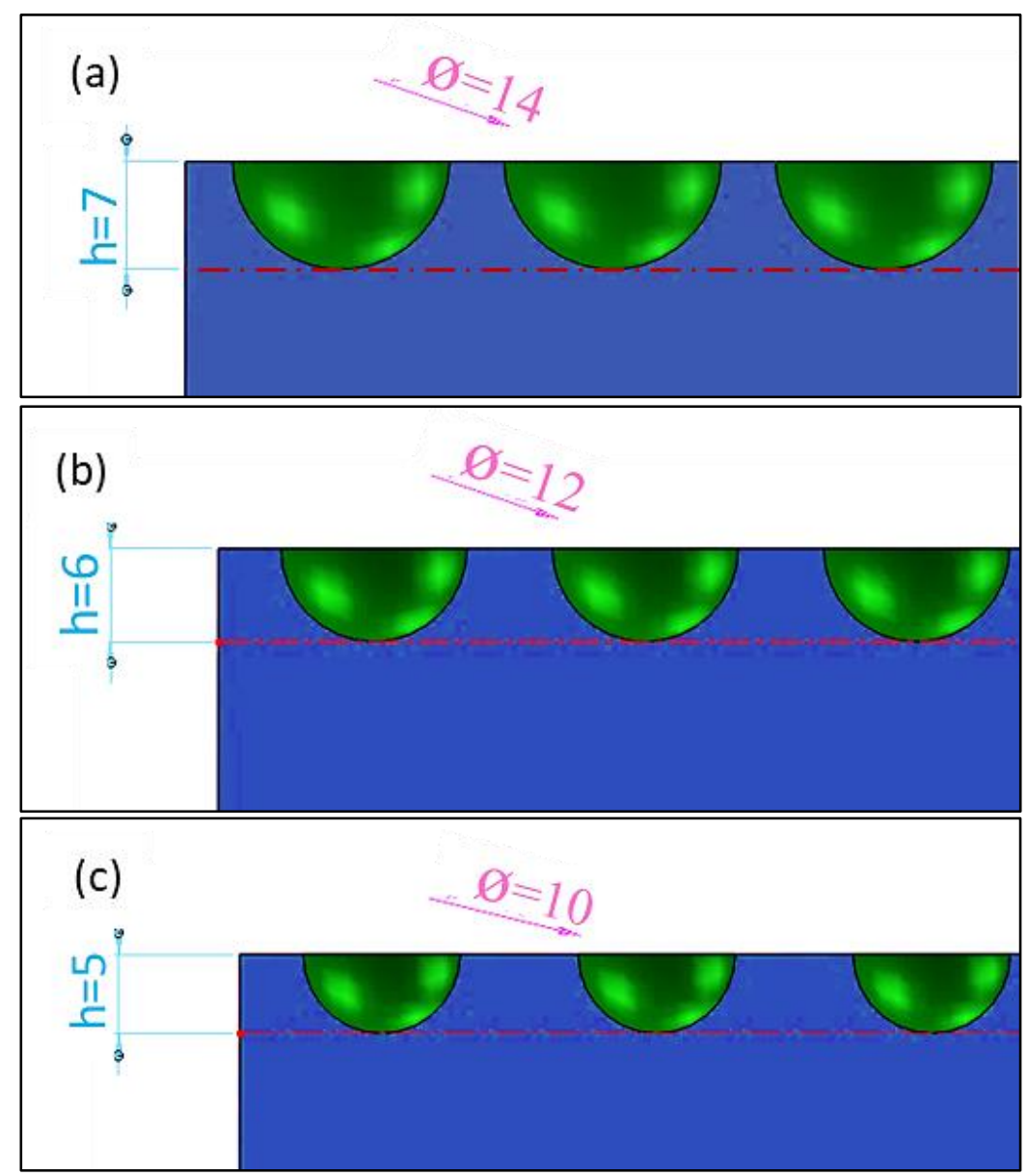

Fig. 4. Cross-sectional view (a) Diameter of $14 \mathrm{~mm}(\mathrm{~b})$ Diameter of $12 \mathrm{~mm}$ (c) Diameter of $10 \mathrm{~mm}$

The plain surface of Aluminium block was also being tested for comparison. The wind tunnel was used to generate uniform airflow at desired speed relative to the work sample under test. The velocity of the air was controlled via an inclined manometer and a calibration. The desired velocity value was set by referring to the slope scale of the inclined manometer value from the calibration graph of the contraction section differential pressure versus velocity graph.

In this experiment, the workpiece was heated up using a Fisher Scientific Isotemp basic hotplate heater. The hot plate heater was used to provide constant and uniform heat energy to the workpiece, where the temperature was set to $60^{\circ} \mathrm{C}$. The temperatures were measured with data acquisition system connected to a personal computer and controlled using PicoLog recorder software. With four channels, the K-Type thermocouples were connected to the workpiece at different locations. Once the velocity was set, the airflow flowed over the workpiece. The temperature of the airflow was measured using an anemometer. The workpiece was allowed to cool down throughout the cooling process until it achieved a room temperature of $25^{\circ} \mathrm{C}$. The temperature drop in the workpiece was measured and recorded at every one-minute interval. The steps were repeated for 17 runs at various conditions. Figure 5 shows the experimental setup in the wind tunnel test section. Then, BoxBehnken of Response Surface Methodology (RSM) was used as the Design of Experiments (DoE) tool to analyse the data. Analysis of Variance (ANOVA) was used to evaluate the relative significance of the input parameters with regards to the cooling time. 


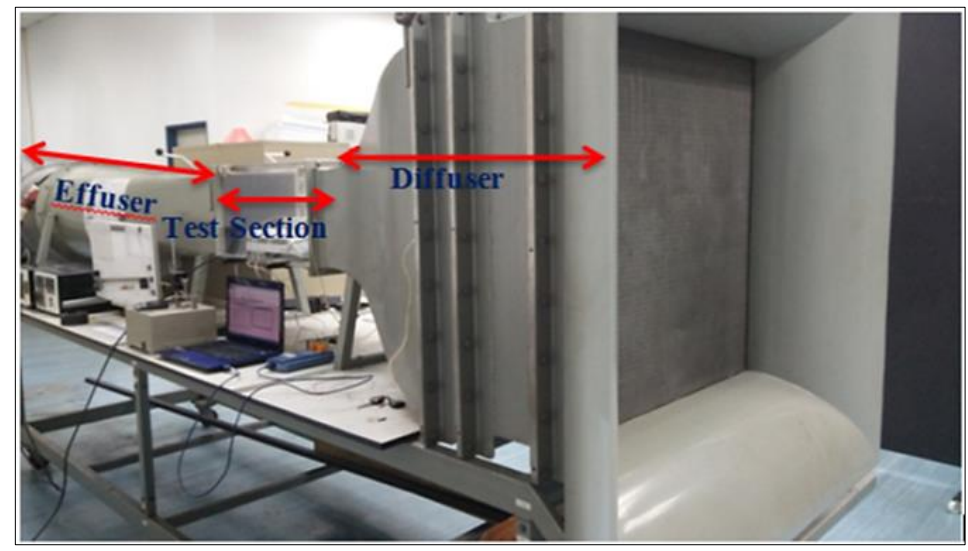

Fig. 5. Experimental setup in the wind tunnel test section

\subsection{Analysis of Computational Fluid Dynamic}

Fluid Dynamics (CFD) was applied to analyze the flow separation inside the dimple profile produced during the cooling process. This method solves non-linear Navier-Stokes equations to simulate a system. The non-linear Navier-Stokes equations comprises continuity, momentum, and energy equations and are known as governing equations. The equations of momentum and continuity were used to determine the velocity vector, whereas the energy equation was used to analyze the distribution of temperature, heat transfer coefficient, and heat transfer rate [25]. The governing equation for continuity, momentum, and energy is denoted as Eq. (1), Eq. (2), and Eq. (3).

Continuity: $\quad \partial \rho / \partial t+\nabla \cdot(\rho \vec{v})=S_{m}$

Momentum: $\quad \partial / \partial \mathrm{t}(\rho \vec{v})+\nabla \cdot(\rho \vec{v} \vec{v})=-\nabla p+\nabla \cdot(\overline{\bar{\tau}})+\rho \vec{g}+\vec{F}$

$$
\text { Energy: } \quad \partial / \partial \mathrm{t}(\rho \mathrm{E})+\nabla \cdot(\vec{v}(\rho \mathrm{E}+\mathrm{p}))=\nabla \cdot\left(\left(\mathrm{K}_{e f f} \nabla T-\sum_{j} h_{j} \overrightarrow{\jmath_{j}}+\left(\overline{\bar{\tau}}_{e f f} \cdot \vec{v}\right)\right)+S_{h}\right.
$$

The first step in CFD analysis is geometry modelling. The dimple profile geometry was drawn using CAD software. Then, the geometry was exported to DesignModeler and the fluid domain was created, which represents the wind tunnel condition. Fluid flow was expected to occur in the fluid domain. CAE Workbench was used to generate the meshing for the dimpled block and fluid domain. Proximity and curvature were used as the size functions for the meshing. The proximity and curvature size functions able to set size range controls at a Global Level.

In the inflation layer, prisms were used to capture the high gradients near the dimpled block. In the inflation layer, the height of the first layer was set to $5 \times 10^{-4}$, and the growth rate was kept at 1.2. Fine relevance was set to ensure a smooth transition of the tetrahedrons from the inflation layer. The total number of layers was 5 . An image of the mesh generation is shown in Figure 6. 


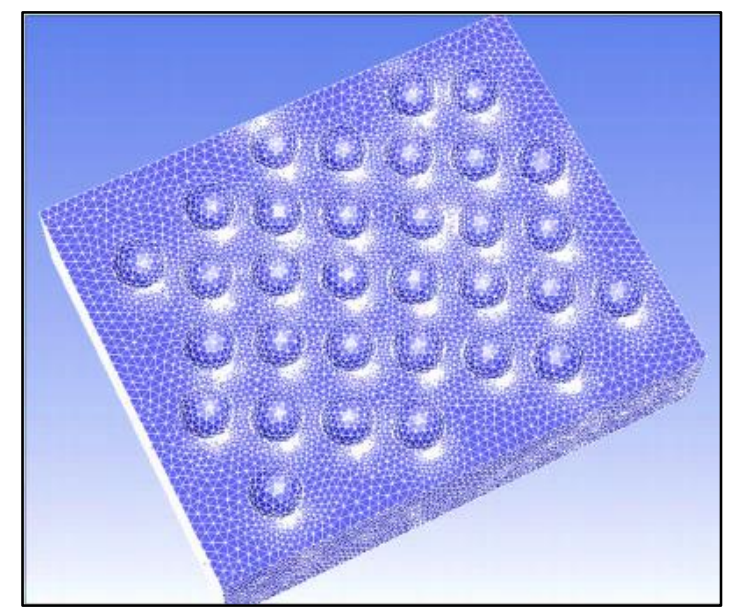

Fig. 6. Mesh generation of the dimpled surface with 283606 mesh elements

In this research, pressure-based solver was selected as it involves laminar flow over a dimpled block. The pressure-based solver is usually applied for comprehensive flow regimes from low-speed incompressible flows. This solver solved energy equations to study the thermal effects on the dimpled block. In addition, the airflow separation or velocity-filled flow was obtained by solving the momentum equation (Eq. (2)). Viscous laminar flow was selected to model the flow conditions. Assigning the boundary condition is a very important step to realistically model the problem. Velocity is another important parameter which is controlled by assigning the velocity value in the inlet of the domain. The fluid in the inlet domain which represent wind tunnel condition in the DesignModeler of CAE Workbench. Figure 7(a) shows the dimple geometry setting in DesignModeler and Figure 7(b) shows the boundary type of velocity inlet, outflow, and wall of the dimple geometry that has been applied in the present study.

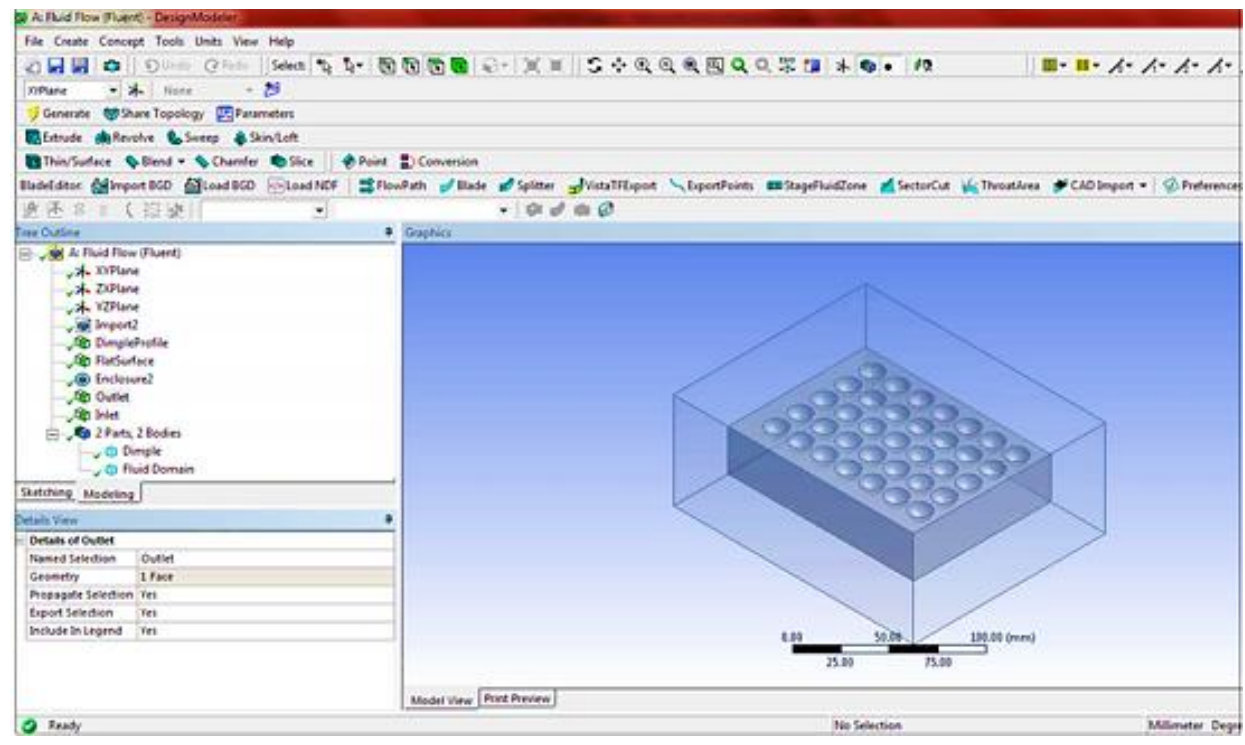

(a) 


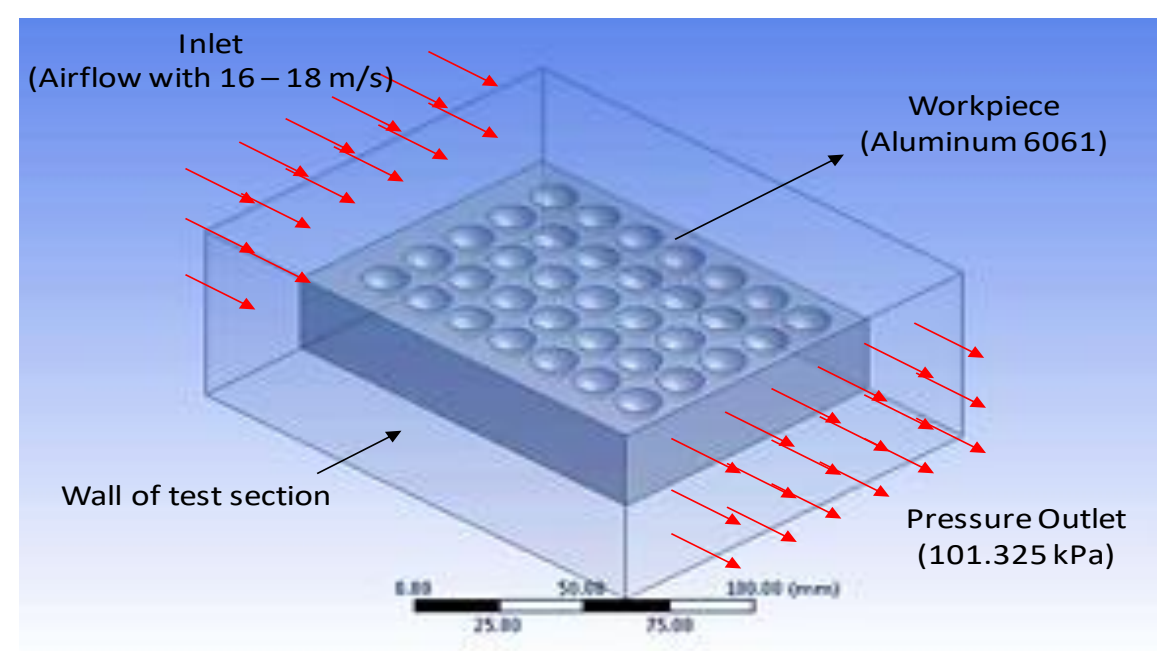

(b)

Fig. 7. Spherical dimples on the Aluminium 6061; (a) Modelling of geometry in DesignModeler (b) Boundary type assigned to the geometries of aluminium workpiece

\section{Results}

\subsection{Experimental Result}

From the experimental results, the longest cooling time of Aluminium was 13.50 min from run 7 and the shortest cooling time was 7.65 min from run 14 as shown in Table 2. From the observation, it shows that the dimple diameter and airflow velocity are significant factors that affect the heat transfer of the block. The dimple with larger diameter took shorter time to transfer heat energy from the dimple block. Therefore, the dimple profile enhanced the heat dissipation of the smooth surface, compared to the plain surface where the cooling temperature recorded was $20.65 \mathrm{~min}$. It shows the maximum dimple block can improve $63 \%$ of cooling time.

Table 2

Cooling time of the spherical dimple block

\begin{tabular}{lllll}
\hline $\begin{array}{l}\text { Experimental } \\
\text { Run }\end{array}$ & $\begin{array}{l}\text { D:Diameter } \\
(\mathrm{mm})\end{array}$ & $\begin{array}{l}\Theta: \text { Angle } \\
\left({ }^{\circ}\right)\end{array}$ & $\begin{array}{l}\text { V:Velocity } \\
(\mathrm{m} / \mathrm{s})\end{array}$ & $\begin{array}{l}\text { Cooling Time } \\
\text { (minutes) }\end{array}$ \\
\hline 1 & 12 & 90 & 16 & 8.26 \\
2 & 12 & 60 & 16 & 10.23 \\
3 & 12 & 90 & 18 & 9.30 \\
4 & 12 & 75 & 17 & 11.02 \\
5 & 14 & 75 & 16 & 10.45 \\
6 & 14 & 75 & 18 & 9.04 \\
7 & 10 & 90 & 17 & 13.50 \\
8 & 12 & 75 & 17 & 12.10 \\
9 & 10 & 75 & 18 & 11.30 \\
10 & 10 & 60 & 17 & 13.35 \\
11 & 14 & 90 & 17 & 12.80 \\
12 & 14 & 60 & 17 & 11.25 \\
13 & 10 & 75 & 16 & 12.50 \\
14 & 12 & 60 & 18 & 7.65 \\
15 & 12 & 75 & 17 & 11.04 \\
16 & 12 & 75 & 17 & 11.82 \\
17 & 12 & 75 & 17 & 12.40 \\
\hline
\end{tabular}




\subsection{Statistical Evaluation}

Using a regression analysis, the quadratic model was found to be significant $(p<0.05)$. Table 3 shows the most significant factor. The most significant factor based on the F-value is interaction velocity ${ }^{2}$ followed by interaction diameter ${ }^{2}$, diameter, interaction angle and velocity, velocity, and interaction angle ${ }^{2}$. The $\mathrm{R}^{2}$ value of 0.96 indicates the regression was well fitted.

Table 3

Analysis of Variance for cooling time

\begin{tabular}{lllll}
\hline Source & F-value & P-Value & Rank & \\
\hline Regression & 14.03 & 0.0011 & & Significant \\
Dia, A & 17.71 & 0.004 & 3 & Significant \\
Angle, B & 0.67 & 0.441 & & \\
Velocity, C & 6.03 & 0.0437 & 5 & Significant \\
Dia $^{2}, \mathrm{~A}^{2}$ & 26.75 & 0.0013 & 2 & Significant \\
Angle $^{2}, \mathrm{~B}^{2}$ & 2.46 & 0.1606 & 6 & Significant \\
Velocity $^{2} \mathrm{C}^{2}$ & 65.68 & $<0.0001$ & 1 & Significant \\
Interaction Dia x Angle $_{\text {Interaction Dia x Velocity }}$ & 1.37 & 0.2796 & & \\
Interaction Angle x Velocity & 0.031 & 0.8654 & & Significant \\
Lack of fit & 9.18 & 0.0191 & 4 & Not significant \\
\hline
\end{tabular}

Figure 8 shows the position of the shortest cooling time. Statistically, the shortest cooling time of $6.99 \mathrm{~min}$ could be achieved when the parameter input was set at: dimple diameter, $\mathrm{D}=13 \mathrm{~mm}$, dimple orientation, $\theta=60^{\circ} \mathrm{mm}$ and airflow velocity, $V=18 \mathrm{~m} / \mathrm{s}$. The following Figure 8-11 show the detailed trends of each input parameter over cooling time. The interaction effect of the dimple diameter and dimple orientation is shown in Figure 8.

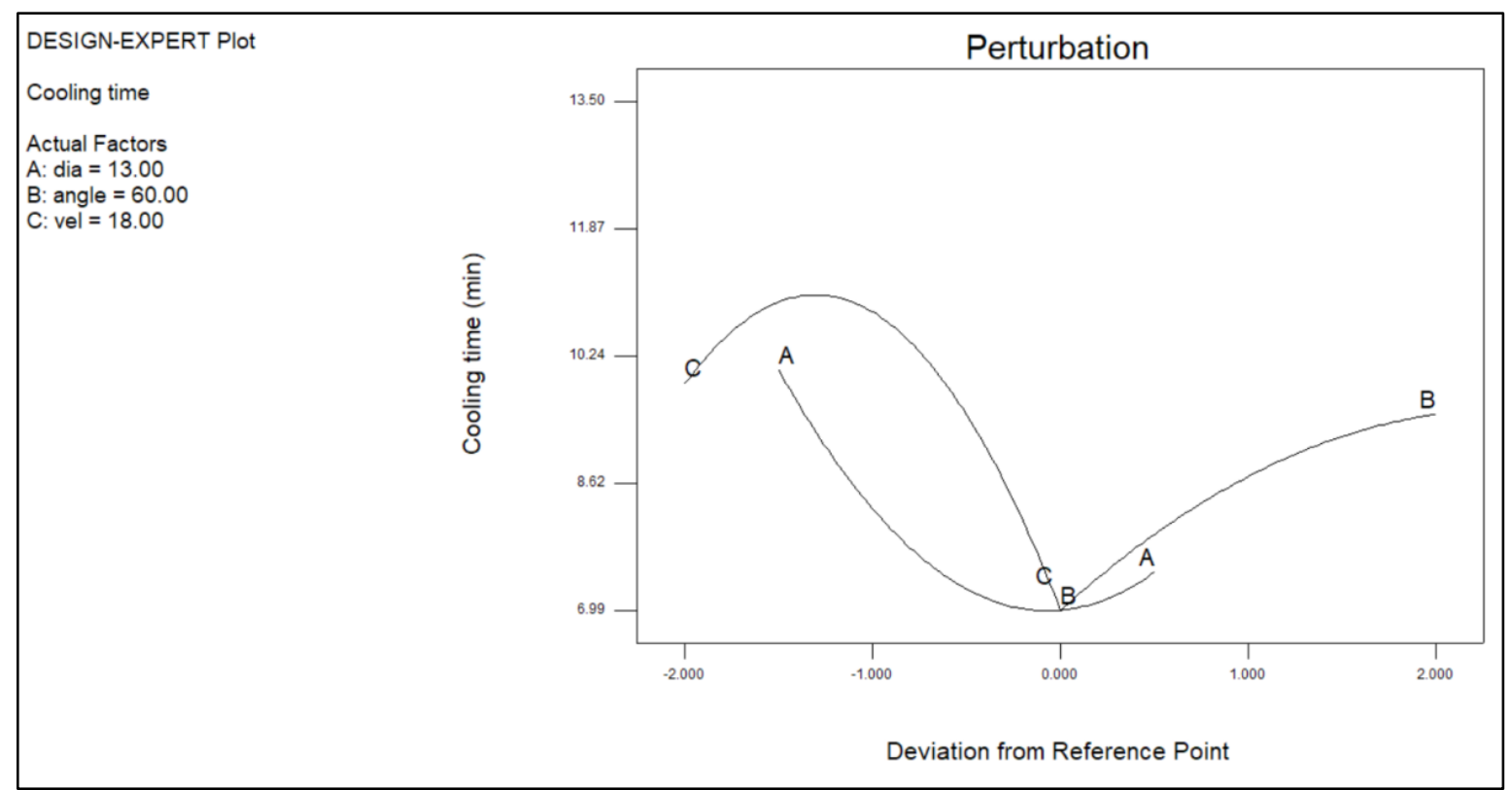

Fig. 8. The setting of parameter input as to achieve shortest cooling

From the graph, it can be observed that the cooling time of the workpiece was faster when the dimple diameter increased. With a dimple diameter of $13 \mathrm{~mm}$, the time taken for the cooling process would be shorter. The cooling time was much better when the air flow velocity $18 \mathrm{~m} / \mathrm{sec}$. 
Figure 9 shows the interaction graph of cooling time over orientation angle. The velocity was set at $18 \mathrm{~m} / \mathrm{sec}$. It can be seen that a staggered arrangement $\left(60^{\circ}\right)$ cooled down faster compared to the workpiece with an inline arrangement $\left(90^{\circ}\right)$. The result was much better when the dimple diameter was $14 \mathrm{~mm}$. For the dimple with inline arrangement, the workpiece took a longer time to dissipate heat energy. At low angles of dimple orientation, the heat transfer coefficient yielded higher values. The phenomena agreed with the findings [21-26].

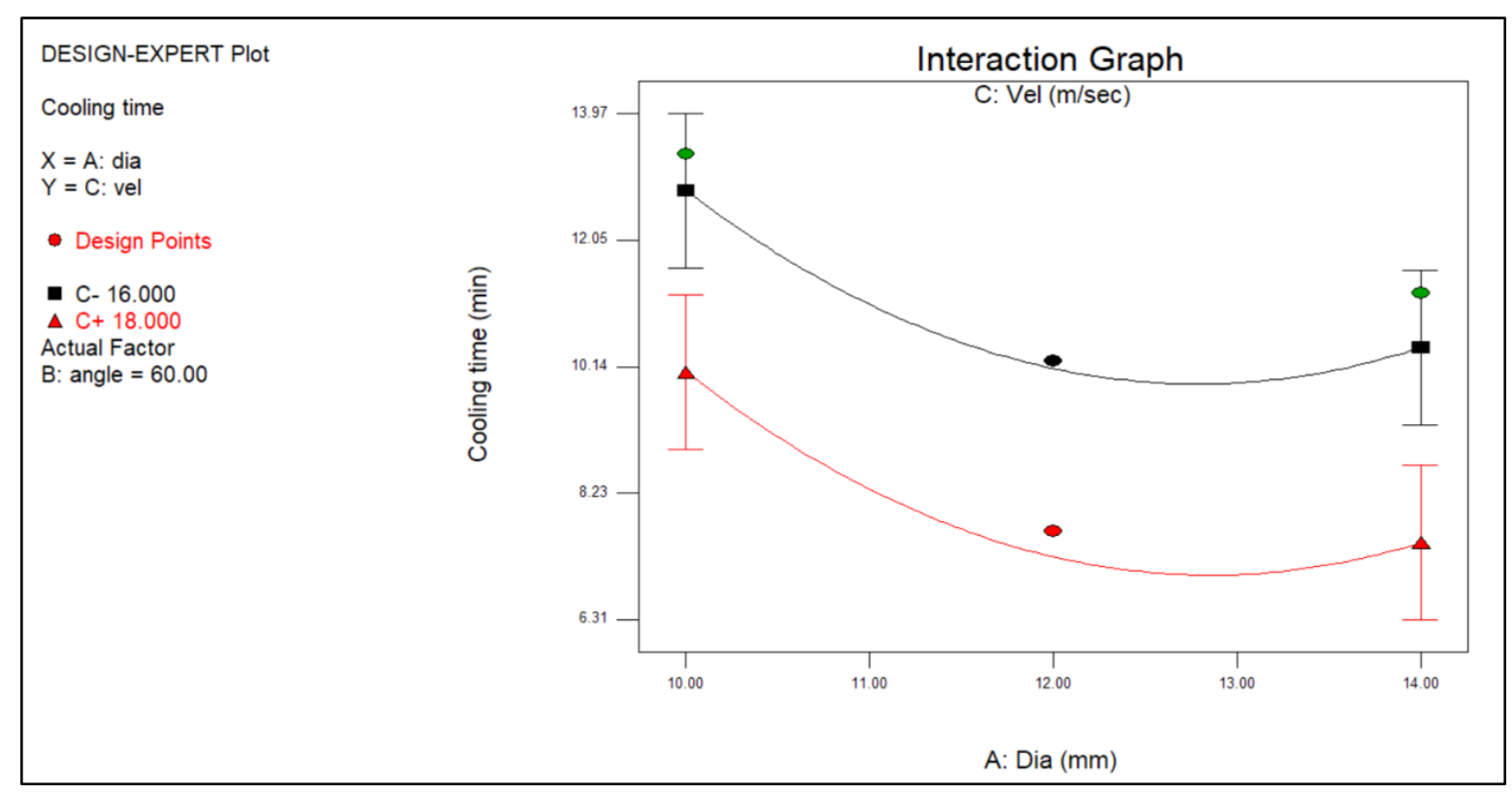

Fig. 9. Graph of cooling time against dimple diameter at dimple orientations of $60^{\circ}$

The relationship between dimple diameter and air velocity with cooling time is illustrated in Figure 10. The slowest cooling time within data set was recorded when the air velocity was around $17 \mathrm{~m} / \mathrm{sec}$. The fastest cooling time was recorded when the air velocity approached $18 \mathrm{~m} / \mathrm{sec}$. Increasing air velocity caused the air particle motion to become more vigorous and rapid. As a result, the collision between air particles and the dimple wall was higher, which stimulated faster heat energy removal. At the same time, vortex formation took place faster.

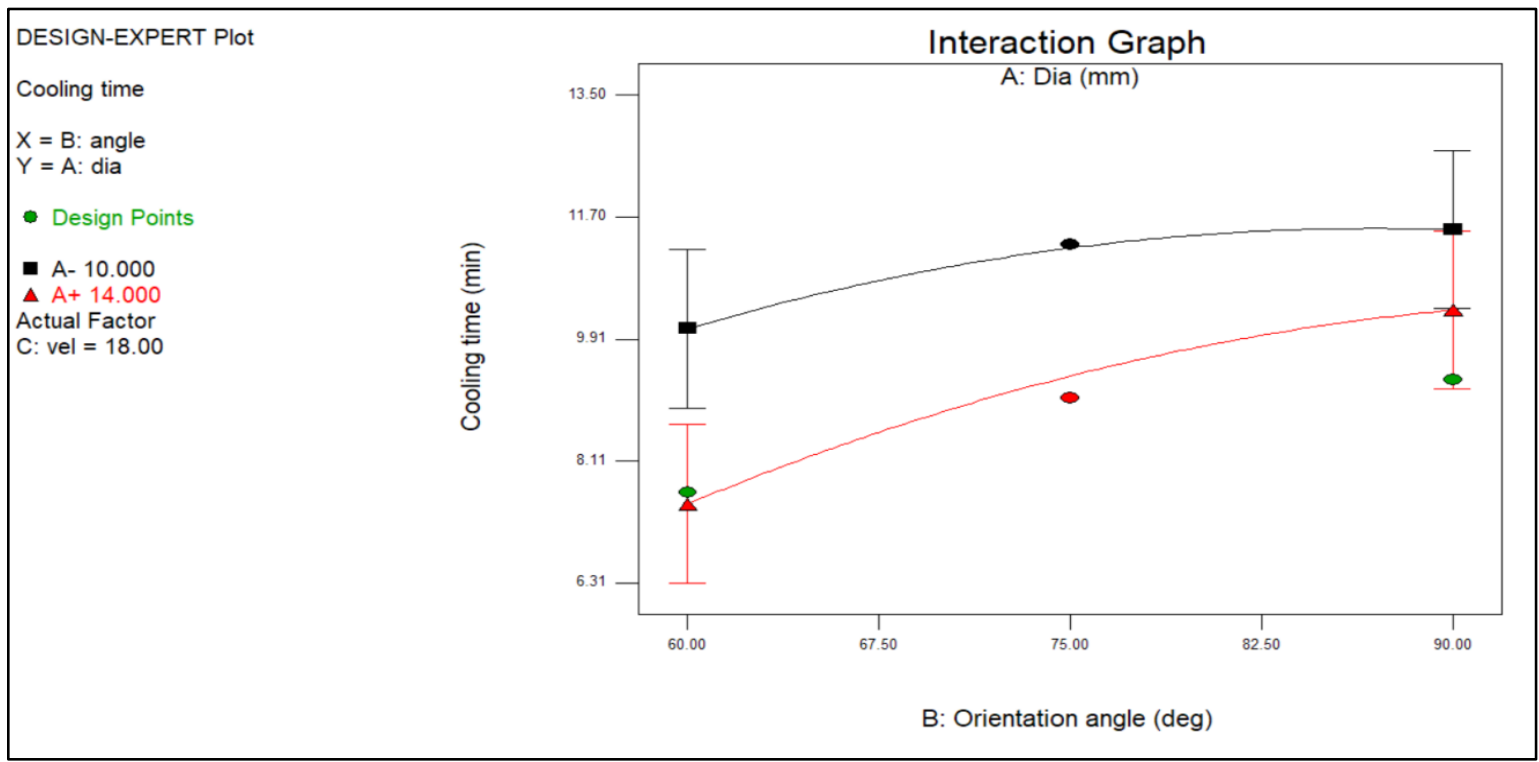

Fig. 10. Graph of cooling time against dimple orientation at different air velocity $18 \mathrm{~m} / \mathrm{sec}$ 


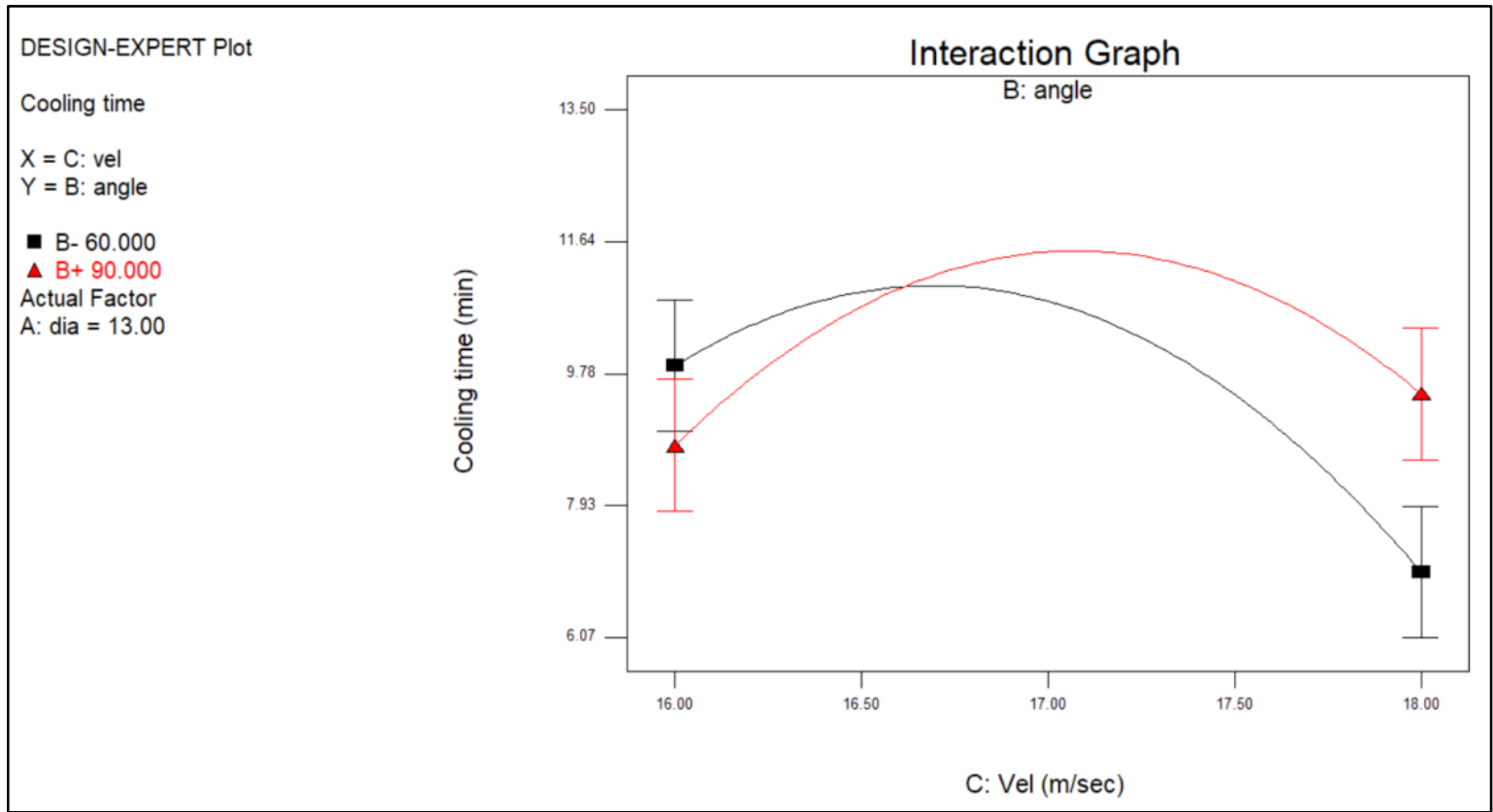

Fig. 11. Graph of cooling time against velocity at dimple diameters of $13 \mathrm{~mm}$

\subsection{Simulation Result}

Throughout the simulation of cooling process, the input heat energy was specified uniformly around the workpiece by setting up the boundary condition. As the air started to flow from the inlet of the workpiece in parallel direction, it could be seen that different velocity magnitudes varied according to the surface profile including wake region behind the dimple. Figure 12 shows the increasing velocity inside the dimple indicating the chaotic flow to create vortex. These dimples induced flow phenomena, therefore heat transfer coefficients increased due to flow reattachment. The heat transfer started to take place, and the dissipation of heat energy from the workpiece is initiated. Figure 13 manifests this phenomenon where the dimple profile is cooler than the flat surface which can be observed from the temperature contour.

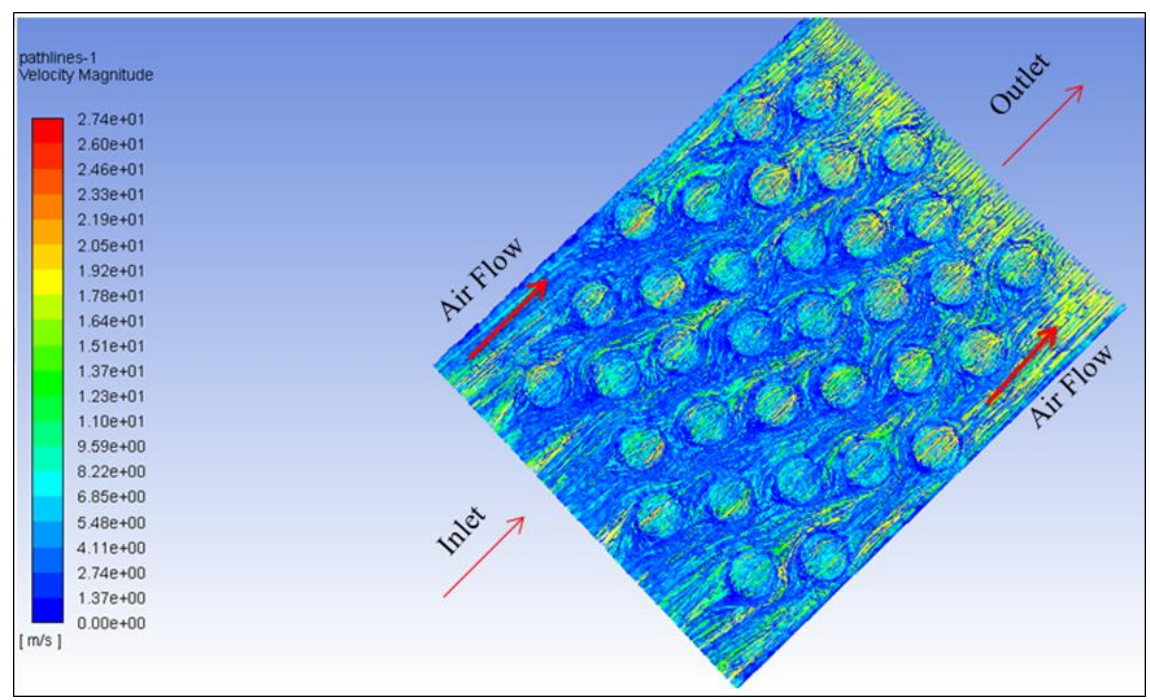

Fig. 12. Simulation of streamline pattern airflow over dimpled surface ( $D=12 \mathrm{~mm}, \theta=60^{\circ}, \mathrm{V}=18 \mathrm{~m} / \mathrm{s}$ ) 


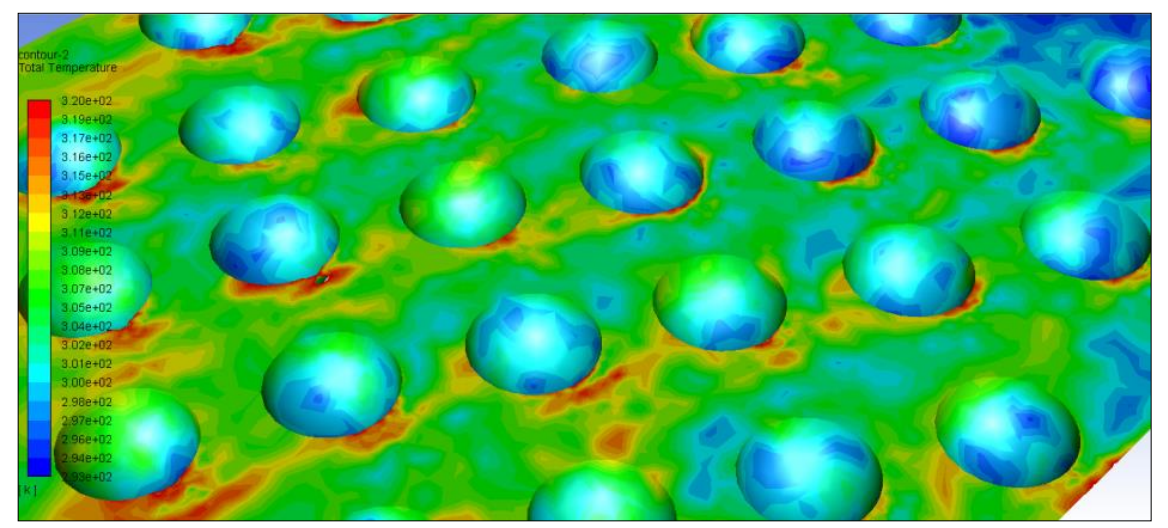

Fig. 13. Visualization of temperature difference on dimpled surface $(D=$ $14 \mathrm{~mm}, \theta=60^{\circ}, V=17 \mathrm{~m} / \mathrm{s}$ )

Based on the experimental data, a total of 14 simulation runs was carried out for different dimple diameters, dimple orientations, and air flow velocities to calculate the Reynolds number, and heat transfer rate. Table 5 shows the result from the simulation.

The graph of heat transfer rate against surface Reynolds number demonstrated the effect of Reynolds number on the total heat transfer rate for the dimpled surface, as shown in Figure 14. It can be observed that the trend line of the graph has positive correlation. The Reynolds number is directly proportional to heat transfer rate. As the Reynolds number increased, the turbulent mixing of airflow also increased, and this resulted in faster heat energy removal from the dimpled surface.

\section{Table 5}

The heat transfer rate and Reynolds number obtained via simulation

\begin{tabular}{llllll}
\hline $\begin{array}{l}\text { No. } \\
\text { Run }\end{array}$ & $\begin{array}{l}\text { Dimple } \\
\text { Diameter,D } \\
(\mathrm{mm})\end{array}$ & $\begin{array}{l}\text { Angle, } \Theta \\
\left({ }^{\circ}\right)\end{array}$ & $\begin{array}{l}\text { Air Velocity,V } \\
(\mathrm{m} / \mathrm{s})\end{array}$ & $\begin{array}{l}\text { Reynolds } \\
\text { Number, Re }\end{array}$ & $\begin{array}{l}\text { Heat } \\
\text { Transfer } \\
\text { Rate, } \mathrm{Q}(\mathrm{W})\end{array}$ \\
\hline 1 & 12 & 90 & 16 & 1640 & 41.03 \\
2 & 12 & 60 & 16 & 1730 & 40.97 \\
3 & 12 & 90 & 18 & 1849 & 41.99 \\
4 & 12 & 75 & 17 & 1776 & 41.54 \\
5 & 14 & 75 & 16 & 1784 & 44.77 \\
6 & 14 & 75 & 18 & 2005 & 46.01 \\
7 & 10 & 90 & 17 & 1605 & 39.22 \\
8 & 12 & 60 & 18 & 1951 & 42.06 \\
9 & 10 & 75 & 18 & 1752 & 40.85 \\
10 & 10 & 60 & 17 & 1746 & 40.30 \\
11 & 14 & 90 & 17 & 1971 & 40.31 \\
12 & 14 & 60 & 17 & 1915 & 44.51 \\
13 & 10 & 75 & 16 & 1540 & 39.68 \\
14 & Flat surface & & & & 11.10 \\
\hline
\end{tabular}




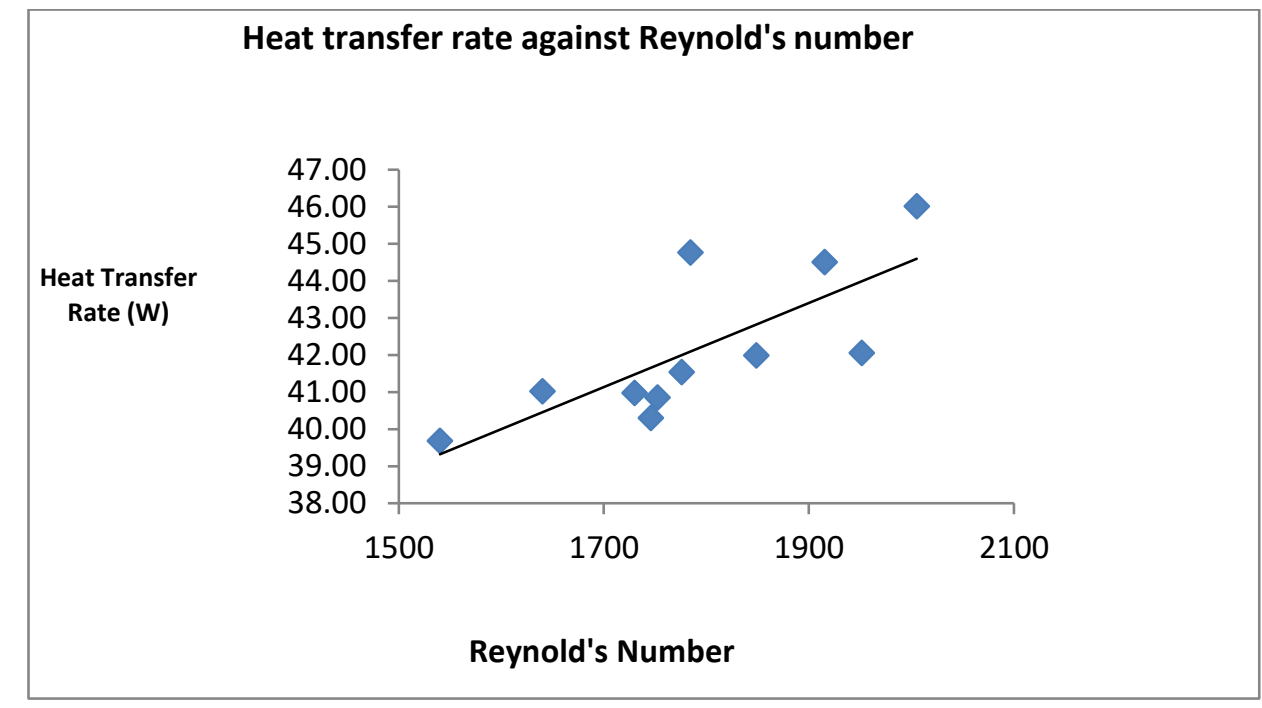

Fig. 14. Graph of heat transfer rate against Reynolds number

Figure 15 shows the correlation between cooling time and heat transfer rate. The cooling time is inversely proportional to heat transfer rate. The cooling time was reduced as the heat transfer rate increased. This is due to the increase in airflow velocity at the inlet, which caused the Reynolds number to become higher. When the airflow velocity increased, the collision between air particles was greater, and this promoted maximum vortex generation [27]. Therefore, the dissipation of heat energy from the dimpled surface was more rapid and the heat transfer rate improved greatly. As a result, the cooling time was decreased.

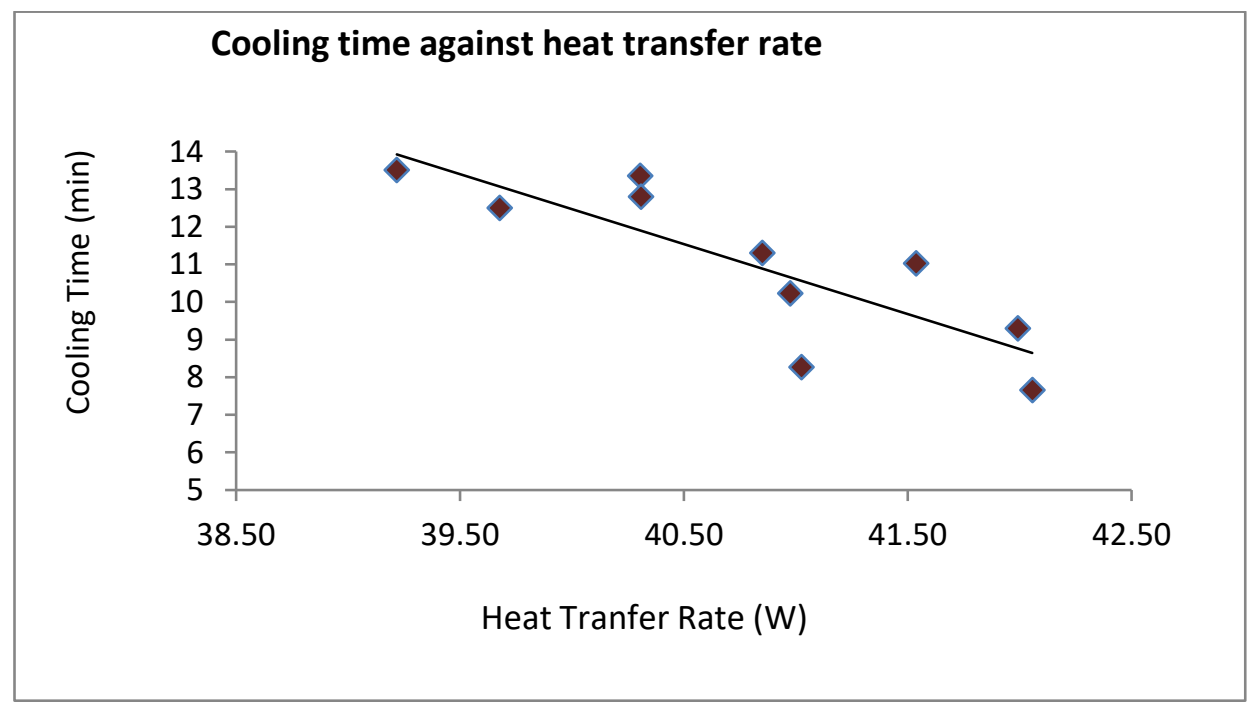

Fig. 15. Graph of heat transfer rate against cooling time

As observed, the air stream followed a straight line as it passed through the upstream zone and started to separate once it came across the dimple profile, as shown in Figure 16. Figure 17 shows the boundary layer of the mainstream, which had separated at the flow separation zone in the dimple. Then, the separated air reattached at the downstream of the dimple profile, namely the reattachment zone, as shown in Figure 18. The reattached airflow then formed a vortex. The airflow in a highly dense dimple profile surface created many vortex formations, which stimulated robust secondary vortex pairs and shear layer reattachment, as illustrated in Figure 19. The huge amount of 
vortex formation in a dimpled surface increased the coefficient of heat transfer. As a result, the dimpled surface took the shortest time to dissipate heat energy from the Aluminium block.

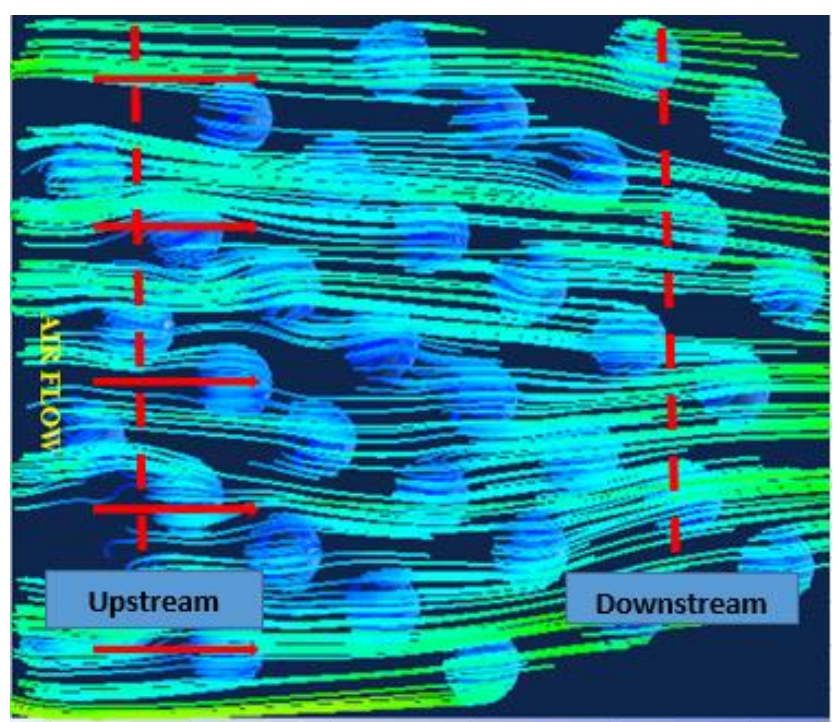

Fig. 16. The airflow structure on a dimpled surface $\left(\mathrm{D}=12 \mathrm{~mm} \theta=60^{\circ} \mathrm{V}=18 \mathrm{~m} / \mathrm{s}\right.$ )

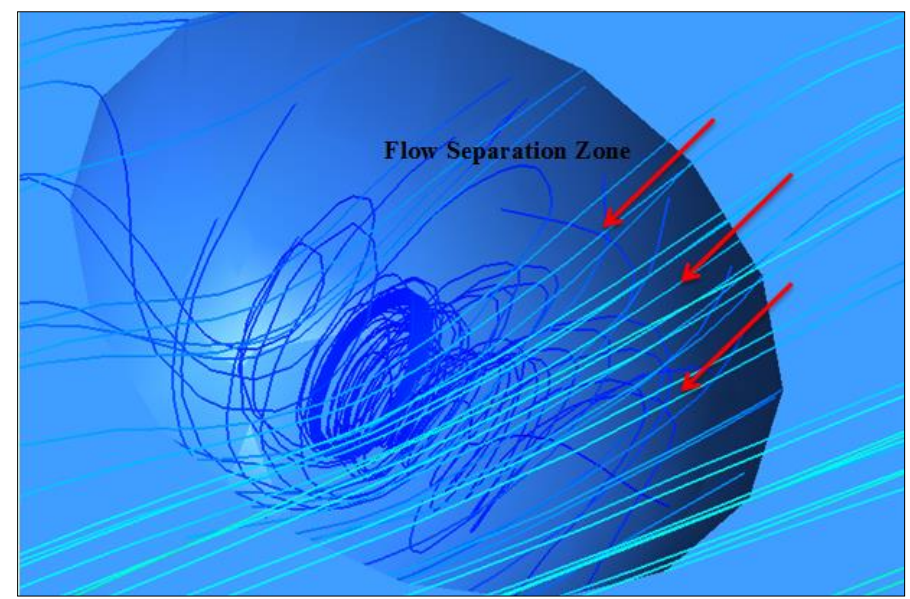

Fig. 17. The formation of the flow separation zone at upstream of the dimple

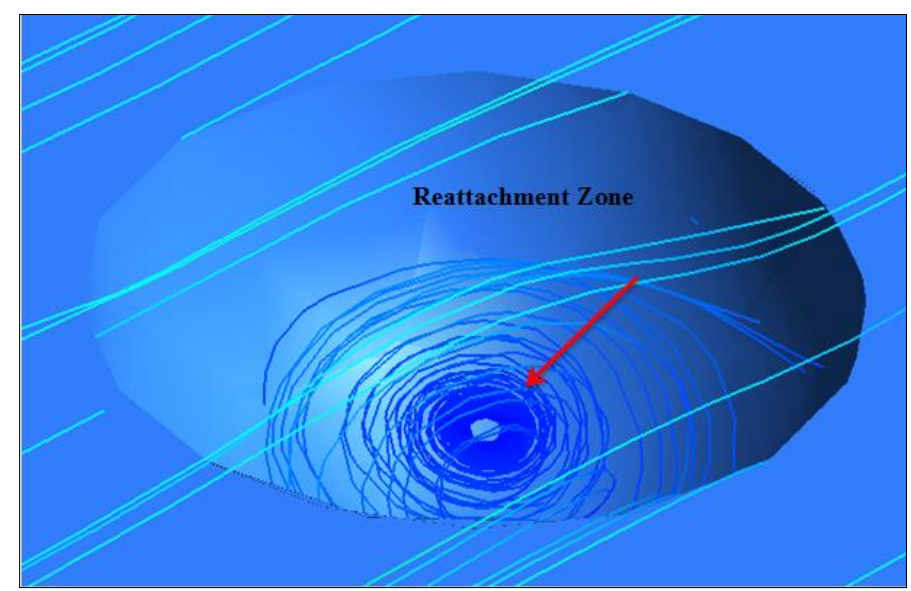

Fig. 18. The separated airflow is reattached at reattachment zone downstream of the dimple 


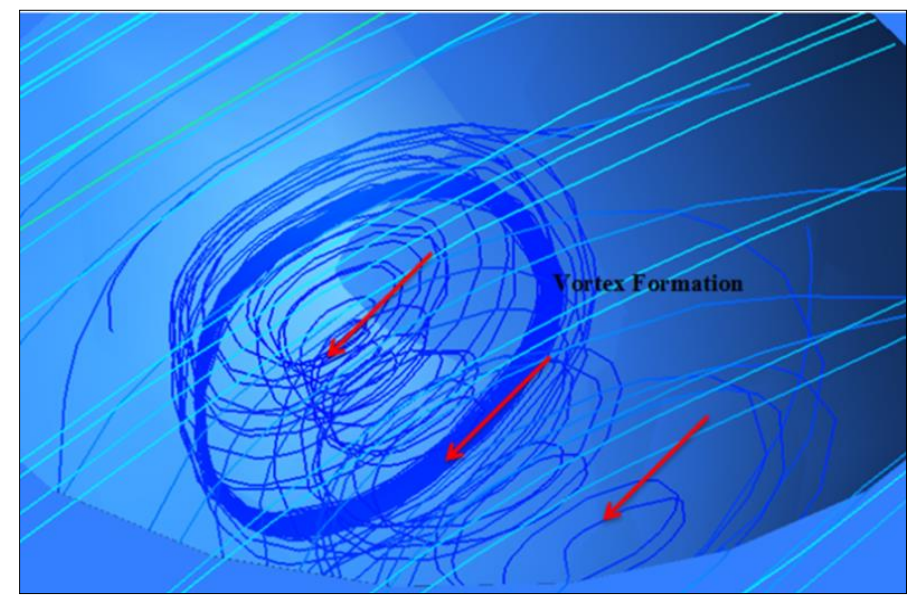

Fig. 19. The reattached airflows to form a vortex structure

\section{Conclusions}

In this article, numerical experiments and simulations had been conducted on the cooling time of dimples characteristic on the flat surface. The main conclusions are summarized as below

i. The cooling time is enhanced when airflow velocity is increased. When the air velocity increases, the air particle motion becomes more vigorous and rapid. Thus, the collision between air particles and the dimple wall is higher, which stimulates faster heat energy removal.

ii. The denser the dimple profile surface, the more vortex formations occur, and more stimulating robust secondary vortex pairs and shear layer reattachment. Increment of heat transfer by the increase of Reynolds number will reduce cooling time. As the Reynolds number increases, the turbulent mixing of airflow also increases, and this resulted in faster heat energy removal from the dimpled surface.

iii. Deeper dimple took a longer time for vortex generation compared to shallower dimples. A surface with a staggered dimple arrangement $\left(60^{\circ}\right)$ dissipates heat energy from the workpiece more easily than the inline dimple arrangement.

iv. On a flat surface, the air stream separation occurred in the upstream zone only, whereas on the dimpled surface, the air stream started to separate at each dimple profile. The mainstream boundary layer was separated at the flow separation zone in a dimple. Then, the separated air reattached downstream of the dimple profile, namely at the reattachment zone. The reattached airflow formed a vortex.

\section{Acknowledgement}

The authors are grateful to the Advanced Manufacturing Center (AMC), Centre of Smart System and Innovative Design (Cossid), Fakulti Kejuruteraan Pembuatan of Universiti Teknikal Malaysia Melaka (JURNAL/2019/AMC/Q00043) for the equipments, tools, and technical support to produce this manuscript. The present work is also supported by Ministry of Higher Education through the grant no: FRGS/2018/FKP-AMC/F00378. 


\section{References}

[1] Bergman, Theodore L., Frank P. Incropera, David P. DeWitt, and Adrienne S. Lavine. Fundamentals of heat and mass transfer. John Wiley \& Sons, 2011.

[2] Sheikholeslami, Mohsen, Mofid Gorji-Bandpy, and Davood Domiri Ganji. "Review of heat transfer enhancement methods: Focus on passive methods using swirl flow devices." Renewable and Sustainable Energy Reviews 49 (2015): 444-469. https://doi.org/10.1016/i.rser.2015.04.113

[3] Sonawane, Tejas, Prafulla Patil, Abhay Chavhan, and B. M. Dusane. "A review on heat transfer enhancement by passive methods." International Research Journal of Engineering and Technology 3, no. 9 (2016): 1567-1574.

[4] Vlachou, Maria C., John S. Lioumbas, and Thodoris D. Karapantsios. "Heat transfer enhancement in boiling over modified surfaces: a critical review." Interfacial Phenomena and Heat Transfer 3, no. 4 (2015). https://doi.org/10.1615/InterfacPhenomHeatTransfer.2016014133

[5] Nadeem Pathan and Sanjay Mitkari. "Heat Transfer Enhancement by Using Dimple Surface." Int. J. Trend Sci. Res. Dev. 2, no. 3 (2018): 2267-2270. https://doi.org/10.31142/ijtsrd12739

[6] Narato, Pathomporn, Makatar Wae-hayee, and Chayut Nuntadusit. "Flow and Heat Transfer Characteristic of Inclined Oval Trench Dimples With Numerical Simulation." CFD Letters 12, no. 11 (2020): 61-71. https://doi.org/10.37934/cfdl.12.11.6171

[7] Sardar, Hasibur Rahman, and Abdul Razak Kaladgi. "Forced convection heat transfer analysis through dimpled surfaces with different arrangements." American Journal of Energy Engineering 3, no. 3 (2015): 37-45. https://doi.org/10.11648/i.ajee.20150303.12

[8] Kuethe, A. "Boundary layer control of flow separation and heat exchange." U.S. Patent 3,741,285, issued June 26, 1973.

[9] Beves, C. C., T. J. Barber, and E. Leonardi. "An investigation of flow over two-dimensional circular cavity." In 15th Australasian Fluid Mechanics Conference, the University of Sydney, Australia, pp. 13-17. 2004.

[10] Paranjape, Abhijit S., Ninad C. Maniar, Deval A. Pandya, and Brian H. Dennis. "Numerical Simulation of Laminar Flow Heat Transfer Enhancement Using Surface Modification." In International Design Engineering Technical Conferences and Computers and Information in Engineering Conference, vol. 46285, p. V01AT02A011. American Society of Mechanical Engineers, 2014. https://doi.org/10.1115/DETC2014-35432

[11] Choi, Jin, Woo-Pyung Jeon, and Haecheon Choi. "Mechanism of drag reduction by dimples on a sphere." Physics of Fluids 18, no. 4 (2006): 041702. https://doi.org/10.1063/1.2191848

[12] Mahmood, G. I., M. L. Hill, D. L. Nelson, P. M. Ligrani, H-K. Moon, and B. Glezer. "Local heat transfer and flow structure on and above a dimpled surface in a channel." J. Turbomach. 123, no. 1 (2001): 115-123. https://doi.org/10.1115/1.1333694

[13] Ghani, J. A., M. N. B. Derani, and W. M. F. Mahmood. "Dimples and heat transfer efficiency." Material Sci \& Eng Int J 1, no. 2 (2018): 2-5. https://doi.org/10.15406/mseij.2018.02.00051

[14] Ligrani, P. M., J. L. Harrison, G. I. Mahmmod, and M. L. Hill. "Flow structure due to dimple depressions on a channel surface." Physics of fluids 13, no. 11 (2001): 3442-3451. https://doi.org/10.1063/1.1404139

[15] Katkhaw, Nopparat, Nat Vorayos, Tanongkiat Kiatsiriroat, Yottana Khunatorn, Damorn Bunturat, and Atipoang Nuntaphan. "Heat transfer behavior of flat plate having 45 ellipsoidal dimpled surfaces." Case Studies in Thermal Engineering 2 (2014): 67-74. https://doi.org/10.1016/i.csite.2013.12.002

[16] Bi, C., G. H. Tang, and W. Q. Tao. "Heat transfer enhancement in mini-channel heat sinks with dimples and cylindrical grooves." Applied Thermal Engineering 55, no. $1-2 \quad$ (2013): $121-132$. https://doi.org/10.1016/i.applthermaleng.2013.03.007

[17] Hu, Jianjun, and Guangqiu Zhang. "Performance improvement of solar air collector based on airflow reorganization: A review." Applied Thermal $\quad$ Engineering $155 \quad$ (2019): https://doi.org/10.1016/i.applthermaleng.2019.04.021

[18] Mahmood, G. I., and P. M. Ligrani. "Heat transfer in a dimpled channel: combined influences of aspect ratio, temperature ratio, Reynolds number, and flow structure." International Journal of Heat and mass transfer 45, no. 10 (2002): 2011-2020. https://doi.org/10.1016/S0017-9310(01)00314-3

[19] Ligrani, P. M., G. I. Mahmood, J. L. Harrison, C. M. Clayton, and D. L. Nelson. "Flow structure and local Nusselt number variations in a channel with dimples and protrusions on opposite walls." International Journal of Heat and Mass Transfer 44, no. 23 (2001): 4413-4425. https://doi.org/10.1016/S0017-9310(01)00101-6

[20] Coy, Edward B., and Stephen A. Danczyz. "Measurements of the effectiveness of concave spherical dimples for enhancement heat transfer." Journal of Propulsion and Power 27, no. 5 (2011): 955-958. https://doi.org/10.2514/1.B34255 
[21] Patil, Pooja, and Padmakar Deshmukh. "An experimental study of heat transfer enhancement in the circular channel with almond shape dimples." IOSR Journal of Mechanical and Civil Engineering (IOSR-JMCE) 11 (2014): 4857. https://doi.org/10.9790/1684-11514857

[22] Chyu, M. K., Y. Yu, H. Ding, J. P. Downs, and F. O. Soechting. "Concavity enhanced heat transfer in an internal cooling passage." In Turbo Expo: Power for Land, Sea, and Air, vol. 78705, p. V003T09A080. American Society of Mechanical Engineers, 1997.

[23] Khan, Amjad, Mohammed Zakir Bellary, Mohammad Ziaullah, and Abdul Razak Kaladgi. "An experimental study on heat transfer enhancement of flat plates using dimples." American Journal of Electrical Power and Energy Systems 4, no. 4 (2015): 34-38. https://doi.org/10.11648/i.epes.20150404.11

[24] Kul'gina, L. M. "Experimental Investigation of Heat Transfer Enhancement by Forced Vibrations." Heat Transfer Research 33, no. $5 \& 6$ (2002). https://doi.org/10.1615/HeatTransRes.v33.i5-6.80

[25] Pandey, Ashish Kumar. "A Computational Fluid Dynamics Study of Fluid Flow and Heat Transfer in a Micro Channel." PhD diss., 2011.

[26] Burgess, N. K., M. M. Oliveira, and P. M. Ligrani. "Nusselt number behavior on deep dimpled surfaces within a channel." J. Heat Transfer 125, no. 1 (2003): 11-18. https://doi.org/10.1115/1.1527904

[27] Alam, AZM Shariful, Md Kamrul Islam, Md Yousuf Mia, and Kazi Afzalur Rahman. "Simulation on heat transfer enhancement in a circular tube for laminar flow with and without insert." In Proceedings of the International Conference on Mechanical Engineering and Renewable Energy, pp. 1-3. 2014. 